\title{
Exploratory modeling and experimental investigation of a vibrating-stripe wind energy converter
}

\author{
Giulio Allesina ${ }^{1}$, Pietro Cingi ${ }^{2}$, Gabriele Gessani ${ }^{1}$, Diego Angeli ${ }^{2 *}$ \\ ${ }^{1}$ Department of Engineering Enzo Ferrari, University of Modena and Reggio Emilia, Via Vivarelli 10, Modena 41125, Italy \\ ${ }^{2}$ Department of Science and Methods for Engineering, University of Modena and Reggio Emilia, Via Amendola 2, Reggio \\ Emilia 42122, Italy
}

Corresponding Author Email: diego.angeli@ unimore.it

https://doi.org/10.18280/ijht.360333

Received: 11 October 2017

Accepted: 17 July 2018

\section{Keywords:}

windbelt, fluttering, modeling, wind energy

\begin{abstract}
The future of portable devices is ultimately a challenge on energy conversion and storage, fought on two fronts: battery life and distributed availability of power sources. Nano-scale energy conversion devices are highly attractive for the market, but the non-PV systems still lack in efficiency and portability. Few years ago the Windbelt ${ }^{\circledR}$ system was presented with a new ingenious rotor-free approach for nanoscale wind energy harvesting through aeroelastic fluttering. Aim of this work is to provide a mathematical model with experimental validation to the scientific framework related to the Windbelt technology. An experimental device with variable length was built and tested under different belt tension conditions. The basis of the windbelt technology consists in the movement of a magnet linked to the belt that oscillates between two coils. In the tested device the movement of the magnet was recorded with a high speed camera and the images processed in order to evaluate instantaneously its rotation and vertical deviation from the resting position. Results of the experimental campaign were compared with the output of an aero-elastic model of the belt. The model is based on the assumption of a belt composed of two interconnected sections: the free belt section, whose aeroelastic behaviour is represented by a nonlinear lift coefficient, and the magnet section, where the motion is governed by the inertial and elastic forces. The results of the testing campaign and the model outputs showed a remarkable agreement for what concerns the frequency response of the system.
\end{abstract}

\section{INTRODUCTION}

Mobile devices and systems are the protagonists of the digital and communication revolution of the latter day. The number of companies all around the world that understand the potential of mobile technology in their businesses is tremendously increasing, in spite of the fact that the great part of these mobile devices has their Achilles' heel in battery life. This issue is pushing the research to develop more performing batteries rather than portable recharge systems, which are less successful [1].

Most of the renewable-energy-based, micro or nanoscale recharging devices on the market have low power productivity, high technology content or high specific costs that affect their effectiveness in terms of portability, scalability and, consequently, proliferation.

The ideal micro-scale recharging/power-production device needs to be as cheap, simple and efficient as possible in order to be suitable in remote applications, furthermore cheap products will be a promising and affordable solutions even for developing countries.

Contrarily to PV systems that are cell-based and intrinsically scalable, wind energy conversion systems have big issues in the downsizing process [2]. Micro scale applications of traditional wind turbines are characterized by low efficiency and high specific costs due to the higher influence of friction in small scale and less advantageous ratio between the rotor and the nacelle areas. For instance, an analysis of micro-rotors reveals that the maximum efficiency achievable is approximately $20 \%$ the ideal Betz limit [3-5].

From preliminary studies, alternative micro-scale applications based on galloping, vortex shedding, or fluttering make no exception, being characterised by even lower efficiencies, if compared to micro-turbines [6-8]. Among these, the so-called "Windbelt ${ }^{\circledR}$ " appears as particularly interesting, as well as less studied and underdeveloped.

Aeroelastic fluttering has been deeply investigated in the past, particularly for its relevance to to the aerospace and civil engineering fields, since, for instance, it might cause catastrophic effects on bridges and airfoils [9-10]. Similar to what happened in the field of thermoacoustics, where the studies about Rijke oscillations suppression in rocket nozzles became the basis for exploiting the phenomena for refrigeration and power production purposes [11-13], aerodynamic oscillations and fluttering began to be exploited in 1981 by Mc Kinney e Delaurier through the creation of a water driven oscillating wingmill-windmill [14]. Moreover, a further historical fact links thermoacoustics and windbelts: behind both these innovative technologies there are the works of Lord Rayleigh. He investigated several resonance and oscillation phenomena, and throughout his career he examined both the aeolian harps and the Rijke oscillations [15-16].

In 2008, twenty-seven years after the work of McKinney and Delaurier, Frayne introduced the Windbelt [17-18]. Contextually, the Windbelt concept was engineered in a range of devices at different scales, by the company Humdinger Wind Energy LLC. These devices were very simple in structure, consisting of a suitably tensioned polymer/fiber 
composite belt or tape, and attached permanent magnets that interacted with adjacent coils. A sketch of the basic device is depicted in Figure 1. This solution involves no rotors nor blades, as it only exploits the oscillations generated on the tape by the wind stream, similarly to an aeolian harp [18-19]. To date, only very scarce and preliminary data from experimentation are available to assess the effectiveness of such devices [6, 20-22], and, to the Authors' knowledge, few works on mathematical modelling has been proposed so far [23-24]. Hence, the development of a simplified model describing the dynamics of windbelts would be beneficial for a more systematic assessment of this kind of energy harvesting devices. Indeed, for micro-scale applications this technology seems to be promising, due to its extreme simplicity and low production costs.

This work investigates experimentally and analytically the fluttering phenomenon, at the basis of the windbelt concept, in a small scale system, with the aim of defining a simple mathematical model to describe the kinematics of the magnet. A coupled dual-belt-sections model is proposed and validated through an experimental campaign, which foresees the construction of a relatively simple prototype and the detection of the magnet position by means of a high speed camera. Results of the video acquisitions were then processed frameby-frame in order to reconstruct the magnet deviation and rotation from the rest position. The acquired data were finally compared with the model outputs, showing promising results.

The prototype used for the experimental comparison had originally been realized with the aim of a preliminary investigation of an optimal configuration in terms of Root Mean Square (RMS) voltage output at the ends of the coil, by testing different belt lengths and tensions. Although the present work is mainly focused on the magnet dynamics, the results of these preliminary analysis are shortly reported, as they proved relevant for choosing convenient values for the belt length and tension for the main study.

\section{EXPERIMENTAL SETUP}

The prototype used, as shown in Figure 1, is composed of: (i) a wooden frame; (ii) a mylar belt, $1.275 \mathrm{~cm}$ wide and with variable length; (iii) two cylindrical neodymium magnets with a mass $\mathrm{m}=2.5 \mathrm{~g}, 6 \mathrm{~mm}$ high and with a diameter $\mathrm{d}=12 \mathrm{~mm}$; (iv) two coils, each consisting of 500 rounds of copper wire (wire diameter $310 \mu \mathrm{m}$ ). While one end of the belt is fastened to the frame, the other is kept at a constant tension by a counterweight. The coils are attached to the frame while the magnet is integrated in the belt. The linear density of the belt has been evaluated by weighing a $30 \mathrm{~m}$ long segment of tape, thus obtaining $\rho=3 \times 10^{-4}[\mathrm{~kg} / \mathrm{m}]$.

The experimental apparatus also includes: (i) two fans, blowing air at a constant average speed $U=2 \mathrm{~m} / \mathrm{s}$; (ii) an oscilloscope connected to the coils to measure the RMS voltage output; (iii) a NanoSense MKIII $1260 \times 1024$ pixels CCD camera by IDT, with a $60 \mathrm{~mm}$ Nikon lens, to track the magnet motion. The acquisition frequency of the camera can range from a maximum of 1000 frames per second $(1 \mathrm{kHz})$, to a minimum of $30(30 \mathrm{~Hz})$; after preliminary testing, an acquisition rate of 250 frames per seconds has been deemed as adequate for the present work.

The subsequent image processing comprised three phases: (i) the recognition of the magnet region within the image; (ii) the determination of the vertical position of the center of mass of the magnet region; (iii) the determination of the modulus of its rotation angle with respect to the longitudinal axis of the belt.

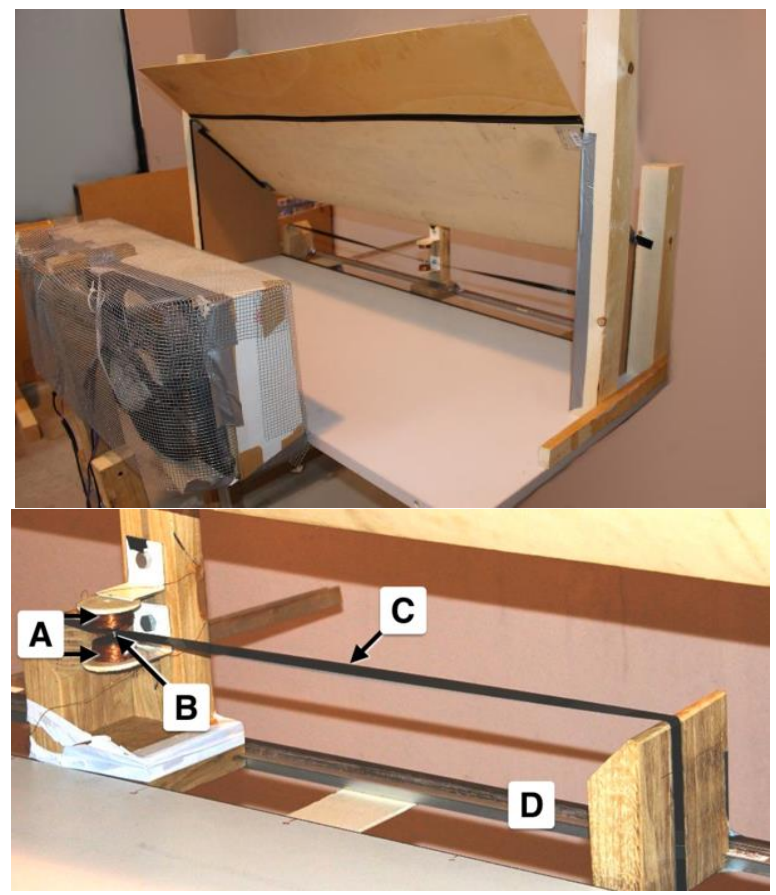

Figure 1. Complete view (top) and detail (bottom) of the experimental apparatus. A: Coils, B: Magnet, C: Tape, D: Frame

\section{MATHEMATICAL MODELING}

In order to analyze the aeroelastic behavior of a generic section of a fluttering windbelt, the dynamical equilibrium between aerodynamic, inertial and elastic forces should be taken into account. To this aim, the widely adopted Typical Section Method, described in [25-26], was deemed as partially unsuitable for the problem at hand. In fact, in first instance, the Typical Section Method, originally devised for airfoils and aircraft wings, assumes an infinite stiffness between adjacent sections, whereas, in the windbelt case, there is almost no bending and torsional stiffness between sections. Secondly, there is the need to take into account the different elastic and aerodynamic forces in the part of the belt which is free to flutter, as well as in the portion which hosts the magnet.

Hence, in the present work, the windbelt device has been described by means of a composite spring-mass system. A simple scheme of the model adopted for a generic section is shown in Figure 2 and described below.

The central part of the belt (which, dynamically, represents the driving part of the system), is modeled as a rigid airfoil with a mass $m$ that is subjected to aerodynamic forces. The motion of the central part could still be described by the equations of the Typical Section theory [25]. In fact, it could be assumed that, in the central region of the belt, the elastic forces generated by the ribbon tension are constant. Furthermore, it can be assumed that no significant aerodynamic forces are generated on the magnet, and, on the other hand, the magnet motion induces no relevant effects on the central area of the ribbon (while, as it will be shown in the following, the opposite effect will be considered to express the motion of the magnet). The dynamic equilibrium of the central 
section is thus expressed by the following set of equations (symbols are defined in the nomenclature section):

$m \ddot{h}+S_{\alpha} \ddot{\alpha}+K_{h} h+q S \frac{\partial C_{L}}{\partial \alpha} \alpha=0$

$I \ddot{\alpha}+S_{\alpha} \ddot{h}+K_{\alpha} \alpha+q e S \frac{\partial C_{L}}{\partial \alpha} \alpha=0$

In the present model, the standard approach is modified as follows. The dynamic effects of unbalanced masses is set to zero because of the symmetry of the section $\left(S_{\alpha}=S_{\alpha} \ddot{h}=\right.$ $S_{\alpha} \ddot{\alpha}=0$ ). Furthermore, due to the strong twist experienced by the belt, the lift coefficient cannot be taken constant with respect to the airfoil rotation $\alpha$. This means that the coefficient of Eqs. (2) and (1) can not be taken as linear. In particular, the lift term was modified as follows:

$L=L(\alpha)=q S \frac{\partial C_{L}}{\partial \alpha} \alpha$ with $|\alpha| \leq 7.0^{\circ}$

$L=L\left(\alpha=7^{\circ}\right)$ with $7.0^{\circ}<|\alpha|<12.0^{\circ}$

$L=0$ with $|\alpha|>12.0^{\circ}$

The effect of the presence of the magnet was taken into account by considering it as a point mass connected to the ground by a pair of springs, and driven by the forces transmitted by the middle belt section. Hence, the magnet dynamic behavior corresponds to the forced vibration motion of a spring-mass system, as described by the following equations:

$m \ddot{h}_{\text {magn }}+\ddot{k}_{h, \text { magn }} h_{\text {magn }}=F_{\text {ext }, h}$

$F_{\text {ext }, h}=k_{h, t r} h$

$I_{\alpha} \ddot{\alpha}_{\text {magn }}+k_{\alpha, \text { magn }} \alpha_{\text {magn }}=M_{\text {ext }, \alpha}$

$M_{\text {ext }, \alpha}=k_{\alpha, t r} \alpha$

where $F_{\text {ext }, h}$ and $M_{\text {ext, } \alpha}$ are the forces transmitted by the driving part of the ribbon. These are obtained by multiplying the displacement of the central part of the ribbon ( $h$ and $\alpha$ ) by the stiffness constant $\left(k_{h, t r}\right.$ and $\left.k_{\alpha, t r}\right)$ of the hypothetical spring that simulates the part of ribbon connecting the magnet and the center.

The elastic forces due to the tensioned ribbon are simulated through two springs that independently connect the center of the airfoil and the magnet to the ground. The transmission of forces between the central section and the magnet section is evaluated through a linear coefficient (i.e. a spring) related to the airfoil section displacement. The stiffness coefficients of the springs used in the modeling are obtained from the geometric analysis of a tensioned ribbon. The details of such an analysis are omitted for the sake of brevity. The relation between angular and vertical displacement and the related opposing force of the ribbon are:

$K_{h}=\frac{F_{h}}{f}=T\left(\frac{l}{-x^{2}+l x}\right)$

$K_{\alpha}=\frac{M_{\alpha}}{\theta} \cong \frac{S^{2}}{12} K_{h}$

where $K_{h}$ is the vertical elastic constant, $K_{\alpha}$ is the torsional elastic constant, and $x, l, S$ are the longitudinal position, length and width of the ribbon, respectively.

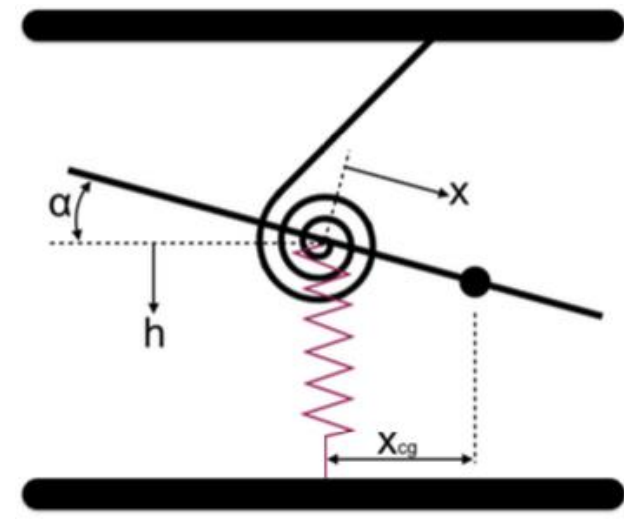

Figure 2. Schematic of the theoretical model of the windbelt device

The set of equations composed by (1), (2), (3), (4) is then integrated by means of standard Runge-Kutta methods.

\section{RESULTS AND DISCUSSION}

\subsection{Preliminary characterization of the device}

A preliminary experimental campaign was devoted to the characterization of the electrical output of the device. Thirty different configurations were investigated. The belt length was varied from 30 to $146 \mathrm{~cm}$ and the tension strength from 0.49 to $2.94 \mathrm{~N}$, as reported in Table 1 .

Table 1. Measured RMS voltage for different values of belt length 1 and applied tension $\mathrm{T}$

\begin{tabular}{|c|c|c|c|c|c|}
\hline $\mathbf{T}$ & $\mathbf{l}=\mathbf{0 . 3 0} \mathbf{~ m}$ & $\mathbf{l}=\mathbf{0 . 6 0} \mathbf{~ m}$ & $\mathbf{l}=\mathbf{0 . 9 0} \mathbf{~ m}$ & $\mathbf{l}=\mathbf{1 . 2 0} \mathbf{~ m}$ & $\mathbf{I}=\mathbf{1 . 4 6} \mathbf{~ m}$ \\
\hline $\mathbf{0 . 4 9} \mathbf{N}$ & 0.0305 & 0.0300 & & & \\
\hline $\mathbf{0 . 9 8} \mathbf{N}$ & 0.0595 & 0.0630 & 0.0688 & 0.0728 & 0.2259 \\
\hline $\mathbf{1 . 4 7} \mathbf{~ N}$ & 0.0603 & 0.1028 & 0.1709 & 0.1039 & 0.3210 \\
\hline $\mathbf{1 . 9 6} \mathbf{N}$ & 0.0626 & 0.1430 & 0.2221 & 0.1590 & 0.3327 \\
\hline $\mathbf{2 . 4 5} \mathbf{N}$ & 0.0553 & 0.1368 & 0.2127 & 0.2317 & 0.3349 \\
\hline $\mathbf{2 . 9 4} \mathbf{N}$ & & & & 0.2459 & 0.3373 \\
\hline
\end{tabular}

The configuration that gave the maximum output was the one with the maximum tension $(2.94 \mathrm{~N}$, corresponding to a mass $m=0.3 \mathrm{~kg}$, applied to the maximum length $(146 \mathrm{~cm})$ of the belt. In this case, an RMS voltage value of $0.34 \mathrm{~V}$ was measured, as reported in Table 1 .

\subsection{Model assessment}

Two further campaigns were then run. The first one used the above described optimal configuration, and was aimed at assessing the analytical model. A second experimental campaign was then lead in order to test the robustness of the model on different sets of parameters (belt lengths and tensions). During these acquisitions the RMS value has been no longer registered, since the aim was then to compare the motion of the magnet with the output of the model.

Figure 3 shows a sequence of snapshots from the CCD acquisitions related to the test at $L=1.46 \mathrm{~m}, m=0.3 \mathrm{~kg}$, 
alongside the extracted signals of the magnet position $h$ and absolute angle of rotation $|\alpha|$. The points corresponding to the snapshots have been highlighted on the diagrams. From the observation of the signals, the oscillatory nature of the phenomenon is clearly observed. The time series of $h$ exhibits a beat phenomenon, typical of vibrations that are combination of sinusoidal signals with similar frequency.
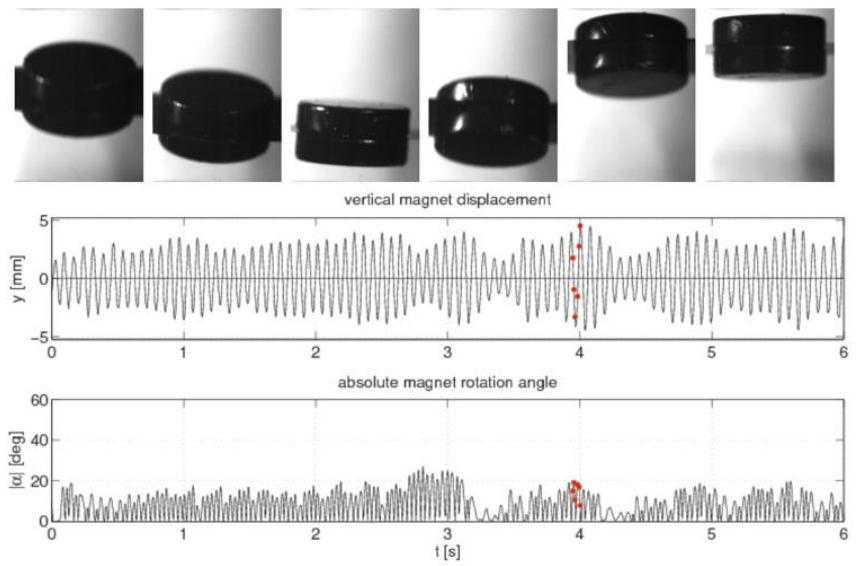

Figure 3. Experimental reconstruction of the magnet motion in terms of $h$ and $\alpha$

Model results for the same configuration are reported in Figure 4. Beats are present in the modeled signal of $h$, and the amplitude of oscillations compares favorably with the experimental result; on the other hand, greater discrepancies are observed for the oscillation angle $\alpha$ : the model predicts much higher rotations than the measured ones.
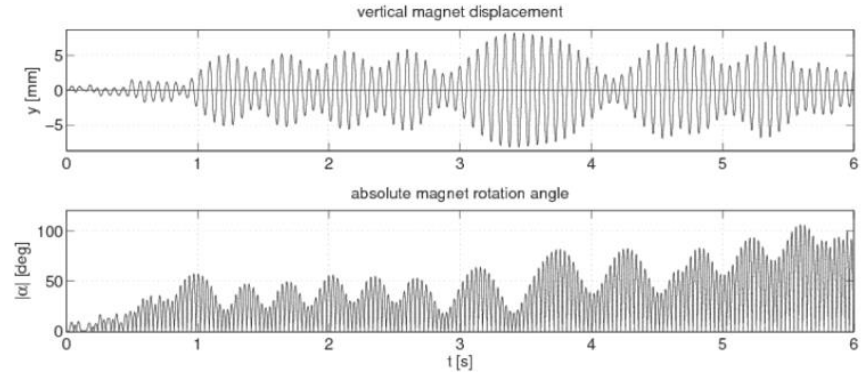

Figure 4. Numerical prediction of the magnet motion in terms of $h$ and $\alpha$

Such a discrepancy is probably due to the oversimplification of the representation of the ribbon. The whole device is indeed divided in only two parts; in order to obtain a more accurate prediction, the system should be split a greater number of elements, each owning two degrees of freedom (i.e. its own $h$ and $\alpha$ ) alongside with their elastic connections to the neighbouring elements. Another factor of discrepancy could be also ascribed to the fact that the rotational elastic constant has been calculated analitically under the hypothesis of small rotation angles, which is not really confirmed by the observations.

A further comparison has been drawn on the normalized frequency spectra of $h$ and $|\alpha|$, see Figure 5. An encouraging agreement is observed between model outputs and experiments. The spectra of $h$ exhibit a clear dominant frequency and a nearby secondary, smaller peak, as a signature of the beat phenomenon. The spectra of $|\alpha|$ instead are characterized by a single dominant frequency (with its halved and second harmonic standing out from the noise, in the experimental spectrum). In both cases, the favorable comparison between the experimental and analytical result, in terms of dominant frequencies, is remarkable.
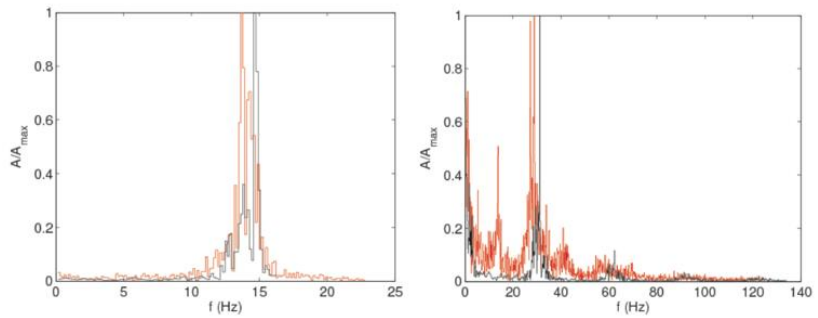

Figure 5. Comparison between the spectrum graphs of real (red) and calculated (black) signals for $L=1.46 \mathrm{~m}$ and $m=0.3$ $\mathrm{kg}$, of $h_{\text {magn }}$ (left) and $\alpha_{\text {magn }}$ (right) respectively

All in all, the concordance between the results in terms of vertical dis- placement suggests that the method lays on the correct bases, and that the the vibrations are indeed influenced by the nonlinearity in the lift coefficient, differently from the classical aeroelasticity theory.

\subsection{Model robustness}

As previously outlined, a further assessment of the model robustness has been carried out, by varying the belt length and tension. The values of $L$ and $T$ adopted are reported in Table 2 , alongside with the absolute error between the measured and calculated fundamental frequency of oscillation of the magnet position $h$. In this phase, the rotation angle $|\alpha|$ has been left aside.

Table 2. Absolute and relative deviation of peak frequencies: experimental vs. modeled belt fluttering

\begin{tabular}{|c|c|c|c|}
\hline$\Delta \mathbf{f}$ & $\mathbf{T}=\mathbf{1 . 9 6} \mathbf{N}$ & $\mathbf{T}=\mathbf{2 . 9 4} \mathbf{~ N}$ & $\mathbf{T}=\mathbf{3 . 9 2} \mathbf{~ N}$ \\
\hline $\mathbf{l}=\mathbf{1 . 4 6} \mathbf{~ m}$ & 1.75 & 0.13 & 0.54 \\
\hline $\mathbf{l = 1 . 1 0} \mathbf{~ m}$ & 1.56 & 0.035 & 0.84 \\
\hline $\mathbf{l = 0 . 9 0} \mathbf{~ m}$ & 0.046 & 2.51 & 0.23 \\
\hline$\Delta \mathbf{f}_{\text {mean }}=$ & \multicolumn{3}{|c}{0.85} \\
\hline$\Delta \mathbf{f}_{\text {mean }}=$ & 3.1 \\
\hline
\end{tabular}
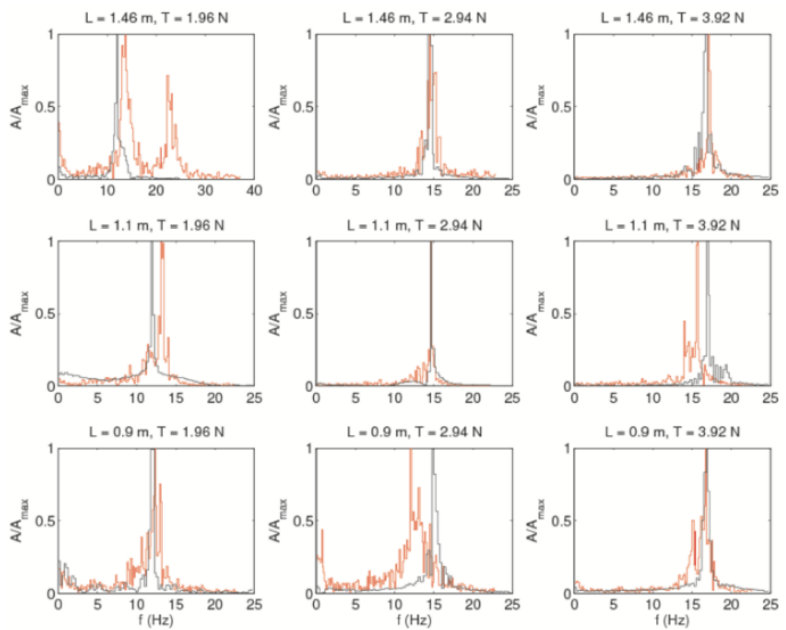

Figure 6. Comparison between the spectra of experimental (red) and calculated (black) $h_{\text {magn }}$ for various configurations 
In Figure 6, the spectra of $h$ for all the tested configurations are shown. It can be observed that the model captures the fundamental oscillation frequency with adequate accuracy in all cases. A maximum relative deviation of approximately $20 \%$ is observed in case $L=0.9 \mathrm{~m}, T=2.94 \mathrm{~N}$, while in the other cases the relative difference always stays below $10 \%$. The mean relative difference results to be $3.1 \%$.

\section{CONCLUSION}

An experimental apparatus for the production on nano-scale of electricity was built and tested. It is based on aeroelastic phenomena, here discussed from the modeling point of view. In fact, a simple model of the system is proposed, tested and discussed in terms of reliability and precision. Its results are compared to the experimental ones showing encouraging accordance.

The robustness of the model is tested through a series of tests where the operation conditions of the real system where varied in compliance with the corresponding analytical models. In almost all the cases the model was proven an effective tool of prediction of the vertical oscillations in the belt.

There is still a long way to go before devices based on aeroelastic oscillations can efficiently be used, but the availability of reliable models is one of the first steps that need to be taken.

\section{REFERENCES}

[1] Lu J, Renjie L, Wang J. (2017). Effect of collaborative utilization of energy storage and wind power on wind power grid-connected and carbon emission. Chemical Engineering Transactions 62: 1117-1122. https://doi.org/10.3303/CET1762187

[2] Wang P. (2018). Design of a fast energy storage and energy conversion system for electric vehicle. Chemical Engineering Transactions 65: 463-468. https://doi.org/10.3303/ CET1865078

[3] Mitcheson PD, Yeatman EM, Kondala GR, Holmes AS, Green TC. (2008). Energy harvesting from human and machine motion for wireless electronic devices. Proceedings of the IEEE 96(9): 1457-1486. https://doi.org/10.1109/JPROC.2008.927494

[4] Villanueva D, Feijoo A. (2010) Wind power distributions: A review of their applications. Renewable and Sustainable Energy Reviews 14: 1490-1495. https://doi.org/ 10.1016/j.rser.2010.01.005

[5] Anghel A, Cardu M. (1992). Preoccupations for wind energy conversion. Energy Conversion and Management 33(12): 1089-1096. https://doi.org/10.1016/01968904(92)90006-I

[6] Briand D, Yeatman E, Roundy S. (2015). Energy harvesting from fluid flows. Wiley-VCH Verlag $\mathrm{GmbH}$ \& Co., KGaA, 297-319.

[7] Siddique ARM, Mahmud S, Van Heyst B. (2015). A comprehensive review on vibration based micro power generators using electromagnetic and piezoelectric transducer mechanism. Energy Conversion and Management 106: 728-747. https://doi.org/ 10.1016/j.enconman.2015.09.071

[8] Sinha S, Chandel SS. (2015). Prospect of solar photovoltaic-micro-wind based hybrid power system in western Himalayan state of Himachal Pradesh in Indial. Energy Conversion and Management 105: 1340-1351. https://doi.org/10.1016/j.enconman.2015.08.078

[9] Scruton C, Flint AR. (1964). Wind-excited oscillation of structures. ICE Proceedings 27(4): 673-702. https://doi.org/10.1680/iicep.1964.10179

[10] Burlibasa A, Ceanga E. (2014). Frequency domain design of gain scheduling control for large wind system in full-load region. Energy Conversion and Management 86: 204-215.

[11] Feldman Jr KT. (1968). Review of the literature on rijke thermoacoustic phenomena. Journal of Sound and Vibration 7(1): 83-89. https://doi.org/10.1016/0022$460 X(68) 90159-4$

[12] Swift G. (2004). What is thermoacoustics. Technical Report LA-UR 04-2298. Los Alamos National Laboratory.

[13] Babei H, Siddiqui K. (2008). Design and optimization of thermoacoustic devices. Energy Conversion and Management 49(12): 3585-3598.

[14] Zhu Q, Haase M, Wu CH. (2009). Modeling the capacity of a novel flow-energy harvester. Applied Mathematical Modelling 33: 2207-2217. https://doi.org/10.1016/j.enconman.2008.07.002

[15] Rayleigh JWS. (1896). The theory of sound part 2. Macmillian.

[16] Rayleigh JWS. (1915). Aeolian tones. Philosophical magazine 29(172).

[17] Humdinger Wind Energy. from https://www.humdingerwind.com

[18] Chen L, Ponta FL, Lago LI. (2011). Perspectives on innovative concepts in wind-power generation. Energy for Sustainable Development 15: 398-410. https://doi.org/10.1016/j.esd.2011.06.006

[19] Rayleigh JWS. (1894). The theory of sound part 1. Macmillian.

[20] Arroyo E, Foong S, Maréchal L, Wood KL. (2014). Experimental study of an omni-directional wind fluttering energy harvester. ASME 2014 Dynamic Systems and Control Conference, DSCC 2014, 3. https://doi.org/10.1115/DSCC2014-5916

[21] Pimentel D, Musilek P, Knight A, Heckenbergerova J. (2010). Characterization of a wind flutter generator. 9th Conference on Environment and Electrical Engineering, EEEIC 2010, pp. 81-84.

[22] Kim SH, Ji CH, Galle P, Herrault F, Wu X, Lee JH, Choi CA, Allen MG. (2009). An electromagnetic energy scavenger from direct airflow. Journal of Micromechanics and Microengineering 19(9). https://doi.org/10.1088/0960-1317/19/9/094010

[23] Aquino AI, Calautit JK, Huges BR. (2017). Integration of aero-elastic belt into the built environment for lowenergy wind harnessing: Current status and a case study. Energy Conversion and Management. https://doi.org/10.1016/j.enconman.2017.03.030

[24] Drachinsky A, Raveh DE. (2016). Limit-cycle oscillations of a pre-tensed membrane strip. Journal of Fluids and Structures 60: 1-22. https://doi.org/10.1016/j.jfluidstructs.2015.06.007

[25] Clark R, Cox D, Curtiss Jr HC, Edwards JW, Hall KC, Peters DA, Scanlan R, Simiu E, Sisto F, Strganac TW. (2005). A Modern Course in Aeroelasticity. Springer.

[26] Anderson JD. (2010). Fundamentals of Aerodynamics. 
Mc-Graw Hill.

\section{NOMENCLATURE}

\section{B}

$\mathrm{C}_{\mathrm{L}}$

$\mathrm{e}$

$\mathrm{K}_{\mathrm{h}}$

$\mathrm{K}_{\alpha}$

h

$\mathrm{I}_{\alpha}$ section width, $m$

lift coefficient

distance between

aerodynamic center of the

section and elastic axis, $m$

Vertical elastic constant, N.m ${ }^{-1}$

Torsional elastic constant, N.m ${ }^{-1}$

spatial displacement, $\mathrm{m}$

moment of inertia of the section, $\mathrm{kg} \cdot \mathrm{m}^{2}$ $\alpha$

$\rho$

$\rho_{\mathrm{a}}$

length of the belt, $\mathrm{m}$

lift, $\mathrm{N}$

mass, $\mathrm{kg}$

dynamic pressure, $\mathrm{Pa}$

static moment of the section, kg.m

surface exposed to the airflow for length unit, $\mathrm{m}$ tension applied to the belt, $\mathrm{N}$ velocity, $\mathrm{m} . \mathrm{s}^{-1}$

\section{Greek symbols}

rotation angle, rad linear density of the tape, kg.m-1

air density, $\mathrm{kg} \cdot \mathrm{m}^{-3}$ 